Bundesgesundheitsbl 2011 · 54:365-371

DOI 10.1007/s00103-010-1235-4

Online publiziert: 23. Februar 2011

(c) Springer-Verlag 2011
C. Poethko-Müller · K. Atzpodien · R. Schmitz · M. Schlaud

Abteilung für Epidemiologie und Gesundheitsberichterstattung, Fachgebiet

Kinder- und Jugendgesundheit, Prävention, Robert Koch-Institut, Berlin

\title{
Impfnebenwirkungen bei Kindern und Jugendlichen. Ergebnisse des Kinder- und Jugendgesundheitssurveys
}

\section{Teil 2: Einflussfaktoren auf elterliche Berichte über Impfnebenwirkungen}

Die umfassende Überwachung der Sicherheit von Impfstoffen beruht heute auf Informationen, die sowohl vor als auch nach der Zulassung eines Impfstoffes gesammelt werden. Da alle Studiendesigns beziehungsweise Überwachungsinstrumente Stärken und Schwächen haben $[1,2,3]$, sollte die Überwachung von Impfnebenwirkungen nach der Zulassung auf Daten aus mehreren Quellen gestützt sein. Standardisierte epidemiologische Studien können die Datenbasis zur Beurteilung von Impfnebenwirkungen ergänzen, und spezielle epidemiologische Studien werden als Bestandteil der Arzneimittelsicherheit nach der Zulassung angesehen [4]. Grundlage für die Datensammlung der meisten Studien oder Surveillancesysteme sind $\mathrm{Pa}$ tientenberichte über mögliche Nebenwirkungen beziehungsweise entsprechende Abfrageergebnisse. Der Wert solcher patientenberichteter Spontanmeldungen von Arzneimittelnebenwirkungen (ADR) wird mittlerweile positiv eingeschätzt [5]. Es gibt aber bisher nur wenig Erkenntnisse darüber, welche persönlichen Faktoren die Wahrscheinlichkeit für eine ADR-Meldung durch einen Patienten beeinflussen [6]. Bei Mitarbeitern im Gesundheitssystem wurde zwar untersucht, welche persönlichen und fachlichen Einflussfaktoren dafür verantwortlich sind, dass ADR-Meldungen unterbleiben [7], jedoch ist wenig darüber bekannt, ob und wie sich solche Aspekte auf Angaben/Berichte zu Impfnebenwirkungen auswirken.

Im vorliegenden Beitrag werden anhand der Daten des Kinder- und Jugendgesundheitssurveys (KiGGS) Prädiktoren für elternberichtete Angaben von Impfnebenwirkungen dargestellt und diskutiert.

\section{Methoden}

Der KiGGS wurde von Mai 2003 bis Mai 2006 vom Robert Koch-Institut (RKI) durchgeführt. Ziel des Befragungs- und Untersuchungssurveys war es, umfassende und bundesweit repräsentative Daten zum Gesundheitszustand von Kindern und Jugendlichen zu erheben. Insgesamt nahmen 17.641 Kinder und Jugendliche (8656 Mädchen, 8985 Jungen) im Alter von o bis 17 Jahren sowie deren Eltern an der Studie teil (Responsequote: 66,6\%), die in 167 Orten Deutschlands zufällig aus den Melderegistern ausgewählt worden waren $[8,9,10]$.

Daten zu soziodemografischen Merkmalen wurden über einen Elternfragebogen erhoben. Aus den Angaben der Eltern zu ihrer Schulbildung und beruflichen Qualifikation, zu ihrer beruflichen Stellung sowie zum Haushaltsnettoeinkommen wurde der Sozialschichtindex nach Winkler [11] bestimmt („hoch“, "mittel“ oder „niedrig“). Kinder und Jugendliche, die selbst aus einem anderen Land zugewandert sind und von denen mindestens ein Elternteil nicht in Deutschland geboren ist oder von denen beide Eltern zugewandert beziehungsweise nicht deutscher Staatsangehörigkeit sind, galten in KiGGS als Migranten. Der Screener auf „Children with Special Health Care Needs" (CSHCN) wurde über den Elternfragebogen erhoben und erfasst über fünf Fragen zum speziellen Versorgungsbedarf bei dauerhaften oder absehbar längerfristig bestehenden körperlichen gesundheitlichen Einschränkungen, Verhaltens- oder Entwicklungsstörungen [12].

Ein standardisiertes, computergestütztes ärztliches Interview (CAPI) mit dem begleitenden Elternteil lieferte Daten zu den Gründen, warum das Kind nicht geimpft wurde, beziehungsweise zu den Nebenwirkungen von Impfungen. Die Untersucher waren aufgefordert, nur schwerwiegende Begleiterscheinungen zu erfassen. Als diesbezügliches Beispiel waren in der Untersucherinstruktion Fieberkrampf, Ödem der gesamten Extremität, generalisierte Urtikaria oder unstillbares Schreien genannt. Die Frage lautete: „Hat Ihr Kind eine Impfung schlecht vertragen?" Wurde die Frage bejaht, wurde um Nennung der Symptome 


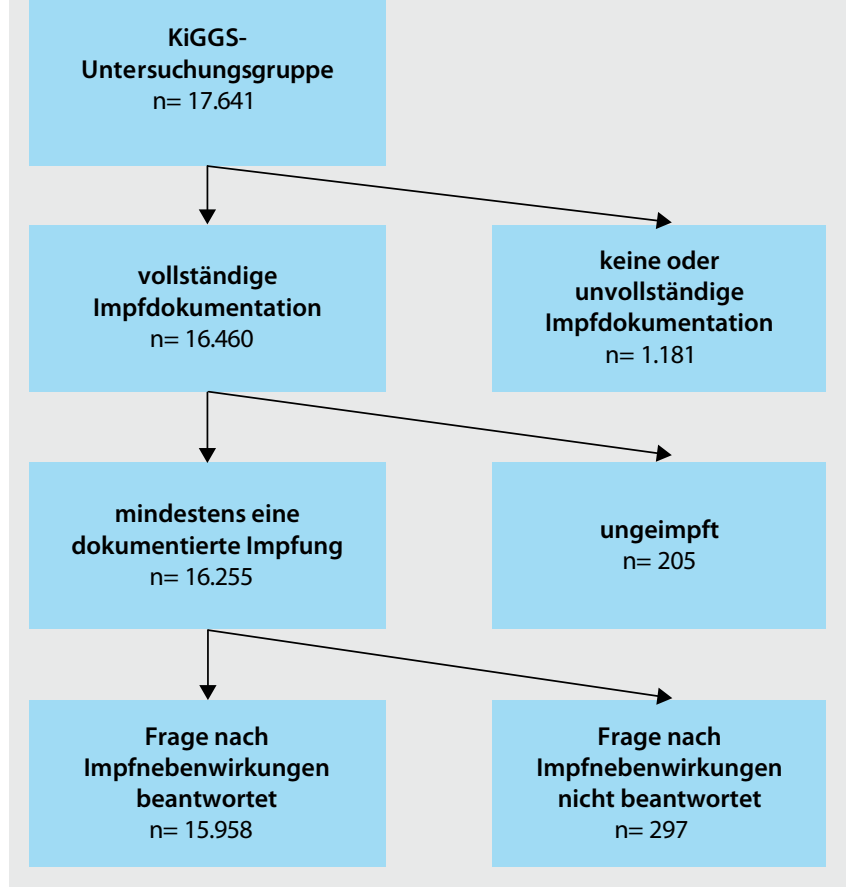

Abb. $1<$ In die Auswertungen eingeschlossene Probanden aus dem Kinder- und Jugendgesundheitssurvey (KiGGS)

und der betreffenden Impfung(en) gebeten und danach gefragt, ob ein Impfschaden anerkannt worden sei. Außerdem wurden die Tage bis zum Symptombeginn erhoben. Die Angabe mehrerer Symptome pro Impfung war möglich, eindeutig redundante Nennungen wurden vor der Datenauswertung bereinigt. Die angegebenen Unverträglichkeiten wurden in der aktuellen MedDRA-Version (Medical Dictionary for Regulatory Activities) als Verdachtsfälle von Nebenwirkungen beziehungsweise Impfkomplikationen entsprechend der international anerkannten Terminologie kodiert und sind im Folgenden als „Impfnebenwirkungen" bezeichnet. Eine Beurteilung des kausalen Zusammenhangs zwischen Impfung und Impfnebenwirkung, wie sie das Paul-Ehrlich-Institut bei Meldungen von Verdachtsfällen auf Impfkomplikationen nach dem Infektionsschutzgesetz (IfSG) vornimmt [13], erfolgte in der KiGGS-Erhebung nicht.

Über die ärztliche Dokumentation in den Impfausweisen wurden Daten zu den angewendeten Impfstoffen und zum Impfzeitpunkt erhoben. Kinder und Jugendliche, für die zum Zeitpunkt der KiGGS-Erhebung keine Impfung dokumentiert war, wurden als ungeimpft definiert. Demgegenüber gelten diejenigen, die laut Impfdokument bis dahin mindestens eine Impfung erhalten hatten [unabhängig davon, ob es sich um eine von der Ständigen Impfkommission (STIKO) empfohlene Standard-, Indikations- oder Reiseimpfung handelte], als geimpft.

Die statistischen Analysen wurden mit der Statistiksoftware SPSS, Version 14, durchgeführt und berücksichtigen das stratifizierte und geclusterte Studiendesign von KiGGS. Berechnet wurden Prävalenzen mit ihren 95\%-Konfidenzintervallen (95\%-KI), die mit Analyseverfahren für komplexe Stichproben bestimmt wurden, um die Korrelation der Probanden innerhalb einer Gemeinde zu berücksichtigen. Mittels uniund multivariater logistischer Regressionsanalysen wurden Determinanten für die Nennung von Impfnebenwirkungen identifiziert und ihre Effektstärken als Odds Ratios (OR mit 95\%-KI) quantifiziert. Die Untersuchung von Determinanten für das Angeben einer Impfnebenwirkung erfolgte hypothesengesteuert durch die Berechnung univariater Odds Ratios für Faktoren, die assoziiert sein könnten mit der Einstellung zu Impfungen (Sozialstatus, Angabe von Gründen für nicht durchgeführte Impfungen, Leben in alten oder neuen Bundesländern), mit der Aufmerksamkeit gegenüber beziehungsweise Bewertung von Gesundheitsstörungen als potenzielle Nebenwirkung einer vorangegangenen Impfung (Sozialstatus, Geschlecht, Anzahl der Geschwister, Migrationshintergrund), mit einer möglicherweise erhöhten Vulnerabilität (Prävalenz von chronischen Gesundheits-, Entwicklungs- oder Verhaltensstörungen mit speziellem Versorgungsbedarf), mit dem zeitlichen $\mathrm{Ab}$ stand zwischen Impfung(en) und Untersuchungstermin (beschränkt auf die Gruppe Masern-Geimpfter) und mit der Anzahl verabreichter Impfungen (Hilfsvariable: Alter). Variablen, die in der univariaten Analyse auf dem 5\%-Niveau statistisch signifikant waren, wurden in das multivariate Modell übernommen. Die Adjustierung für mögliche Zusammenhänge mit dem Geschlecht der Kinder wurde aus grundsätzlichen Erwägungen beibehalten, obwohl Geschlecht in der univariaten Auswertung nicht statistisch signifikant mit der Wahrscheinlichkeit für die Angabe von Impfnebenwirkungen assoziiert war. Gruppenunterschiede mit 95\%-KI, die sich nicht überschneiden, wurden als statistisch signifikant bewertet. Um repräsentative Aussagen treffen zu können, wurden die Analysen mit einem Gewichtungsfaktor durchgeführt, der Abweichungen der KiGGS-Teilnehmer von der Bevölkerungsstruktur der o- bis 17-Jährigen (Stand: 31.12.2004) hinsichtlich Alter, Geschlecht, Region (Ost/ West/Berlin) und Staatsangehörigkeit korrigiert.

\section{Ergebnisse}

Von den insgesamt 17.641 KiGGS-Probanden lagen für 16.460 (93,3\%) Kinder und Jugendliche ausreichende Daten zur Beurteilung des Impfstatus vor. Bei 16.255 Kindern und Jugendlichen war wenigstens eine Impfung im Impfpass dokumentiert. Für 15.958 Kinder und Jugendliche (90,5\% aller Untersuchten) wurde auch die Frage nach Impfnebenwirkungen beantwortet. Diese Gruppe wurde in die folgenden Auswertungen eingeschlos$\operatorname{sen}(\bullet$ Abb. 1).

Die nicht in die Auswertungen eingeschlossene Gruppe unterschied sich in den Merkmalen Alter, Schicht, Alter der Mutter, Migrationsstatus und in der elterlichen Einstellung zu Impfungen von 
der ausgewerteten Gruppe (• Tab. 1): Jüngere Kinder und Kinder, deren Eltern Vorbehalte gegen Impfungen äußerten, konnten häufiger deshalb nicht in die Untersuchung eingeschlossen werden, weil sie ungeimpft waren, während Jugendliche, Migranten und Kindern aus Familien mit niedrigem sozioökonomischem Status häufiger deshalb nicht in die untersuchte Gruppe eingeschlossen werden konnten, weil die Impfdokumentation nicht vorgelegt worden war. Insgesamt betrug der Anteil der Probanden, die nicht in die folgenden Auswertungen eingeschlossen werden konnten, 9,5\%. Die Unterschiede zwischen der in die Analysen eingeschlossenen Gruppe und der KiGGS-Gesamtgruppe waren gering.

Eltern von 332 [2,1\% (95\%-KI 1,8-2,5)] Kindern und Jugendlichen gaben an, dass nach einer oder mehreren Impfungen Unverträglichkeiten aufgetreten waren. Schlecht vertragene Impfungen wurden mit 2,4\% (95\%-KI 1,9-3,0; $\mathrm{n}=193)$ tendenziell etwas häufiger für Jungen angegeben als für Mädchen (1,9\%; 95\%-KI 1,5$2,3 ; n=144)$. Bedeutsame Geschlechtsunterschiede mit Blick auf die Häufigkeit einzelner schlecht vertragener Impfungen lagen nicht vor (Daten nicht gezeigt). Für sieben- bis zehnjährige Kinder wurden häufiger Impfnebenwirkungen angegeben $(2,8 \%$; $95 \%$-KI 2,2-3,6) als für drei- bis sechsjährige Kinder $(1,4 \%$; 95\%-KI 1,0-2,0). Impfnebenwirkungen wurden häufiger berichtet für in den alten Bundesländern lebende Kinder, für Kinder aus Familien mit einem hohen sozioökonomischen Status, für Kinder ohne Migrationshintergrund und für Kinder mit einem positiven CSHCN-Screener (• Tab. 2). Am häufigsten wurden Impfnebenwirkungen für Kinder angegeben, deren Eltern es für besser hielten, dass das Kind (einzelne) Erkrankungen selbst durchmacht (6,9\%; 95\%-KI 5,1-9,2 versus 1,9\%; 95\%-KI 1,5-2,2).

Das Geschlecht des Kindes und Variablen, die sich in der univariaten Auswertung auf dem 5\%-Niveau als statistisch signifikant erwiesen, wurde in ein multivariates logistisches Regressionsmodell aufgenommen. Das Leben in den alten Bundesländern, ein positiver CSHCN-Screener und eine kritische elterliche Impfeinstellung waren unab-

Bundesgesundheitsbl 2011 · 54:365-371 DOI 10.1007/s00103-010-1235-4

(c) Springer-Verlag 2011

\section{Poethko-Müller $\cdot$ K. Atzpodien $\cdot$ R. Schmitz $\cdot$ M. Schlaud}

Impfnebenwirkungen bei Kindern und Jugendlichen. Ergebnisse des Kinder- und Jugendgesundheitssurveys. Teil 2: Einflussfaktoren auf elterliche Berichte über Impfnebenwirkungen

\section{Zusammenfassung}

Die Studiendesigns und Instrumente zur Überwachung der Impfstoffsicherheit haben alle Stärken und Schwächen, sodass zu diesem Zweck Daten aus mehreren Quellen genutzt werden sollten. Grundlage für entsprechende Datensammlungen sind unter anderem Selbstangaben von Patienten über mögliche Nebenwirkungen. Bisher ist aber wenig über die Faktoren bekannt, die beeinflussen, ob Personen Impfnebenwirkungen angeben beziehungsweise berichten. Von 2003 bis 2006 wurden im repräsentativen Kinderund Jugendgesundheitssurvey (KiGGS) von insgesamt 17.641 Probanden im Alter bis zu 17 Jahren Daten zu soziodemografischen Merkmalen, verabreichten Impfstoffen, zum Impfzeitpunkt und zu schlecht vertragenen Impfungen erhoben und Prädiktoren für elternberichtete Impfnebenwirkungen durch uni- und multivariate Analysen ermittelt. Le- ben in den alten Bundesländern (OR 1,61; 95\%-KI 1,08-2,39), ein positiver CSHCN (Children with Special Health Care Needs)-Screener (OR 1,49; 95\%-KI 1,08-2,04) und eine kritische elterliche Impfeinstellung (OR 3,29; $95 \%-K I 2,28-4,75$ ) waren unabhängig voneinander mit einer höheren Wahrscheinlichkeit verbunden, dass Eltern Impfnebenwirkungen in der Befragung berichteten. Die Ergebnisse deuten darauf hin, dass die elterliche Wahrnehmung und Bewertung von Impfnebenwirkungen sowie ihre allgemeine Einstellung zu Impfungen die Angaben zu möglichen Nebenwirkungen beeinflussen könnte.

Schlüsselwörter Gesundheitssurvey · Kinder · Jugendliche . Impfungen · Nebenwirkungen · KiGGS

\section{Suspected adverse reactions after vaccination. Results from the German Health Interview and Examination Survey for Children and Adolescents. Part 2: predictors of parental reporting of suspected adverse reactions after vaccinations}

\section{Abstract}

Each method to monitor vaccine safety has strengths and limitations. Therefore, vaccine safety monitoring should rely on different types of data sources. Methods commonly rely on patient-reported adverse reactions. Little is, however, known about factors that may affect the probability with which patients report adverse reactions to vaccines. From 2003-2006, the representative National Health Interview and Examination Survey for Children and Adolescents ("Kinderund Jugendgesundheitssurvey", KiGGS) retrospectively collected information about vaccines, vaccination dates, and suspected vaccine related adverse reactions from a total of 17,641 participants ( $<17$ years). Poorly tolerated vaccinations were more likely reported from parents living in former West Germany compared to former East Germany (OR 1.61; $95 \% \mathrm{Cl} 1.08-2.39$ ), parents of children with special health care needs (OR $1.49 ; 95 \% \mathrm{Cl}$ 1.08-2.04), and from parents reporting reservations against vaccinations (OR $3.29 ; 95 \% \mathrm{Cl}$ 2.28-4.75). Parental reporting of adverse vaccine reactions appears to be associated with parental perception and assessment of possible adverse vaccine reactions, as well as with the parents' attitude towards immunization in general.

\section{Keywords}

Health surveys - Children · Adolescents . Vaccination - Suspected adverse reactions . KiGGS 
Tab. 1 Soziodemografische Merkmale der ausgewerteten, nicht auswertbaren und der KiGGS-Gesamtgruppe

\begin{tabular}{|c|c|c|c|c|}
\hline & KiGGS gesamt & $\begin{array}{l}\text { Ausgewertete } \\
\text { Gruppe }\end{array}$ & $\begin{array}{l}\text { Nicht auswertbare } \\
\text { Gruppe }\end{array}$ & $\mathrm{p}$-Wert ${ }^{\mathrm{a}}$ \\
\hline & $\%(95 \%-K I)$ & $\%(95 \%-K I)$ & $\%(95 \%-K I)$ & \\
\hline Geschlecht & & & & 0,264 \\
\hline Mädchen & $48,7(48,5-49,9)$ & $48,8(48,5-49,2)$ & $47,3(44,9-49,8)$ & \\
\hline Junge & $51,3(51,1-51,5)$ & $51,2(50,8-51,5)$ & $52,7(50,2-55,1)$ & \\
\hline Alter & & & & $<0,001$ \\
\hline $14-17$ & $26,3(25,7-26,9)$ & $25,1(24,3-25,8)$ & $38,2(35,7-40,7)$ & \\
\hline $11-13$ & $17,3(17,0-17,6)$ & $17,0(16,6-17,4)$ & $20,2(18,1-22,4)$ & \\
\hline $7-10$ & $21,7(21,4-22,0)$ & $22,2(21,8-22,6)$ & $17,4(15,5-19,4)$ & \\
\hline $3-6$ & $21,0(20,8-21,3)$ & $21,9(21,5-22,3)$ & $13,5(11,8-15,5)$ & \\
\hline $0-2$ & $13,6(13,3-13,9)$ & $13,9(13,5-14,3)$ & $10,7(9,2-12,5)$ & \\
\hline Region & & & & 0,365 \\
\hline Ost & $16,8(12,6-22,2)$ & $17,1(12,7-22,5)$ & $15,0(9,9-22,0)$ & \\
\hline West & $83,2(77,8-87,4)$ & $82,9(77,5-87,3)$ & $85,0(78,0-90,1)$ & \\
\hline Sozialstatus & & & & $<0,001$ \\
\hline Niedrig & $27,5(26,1-28,9)$ & $26,6(25,2-27,9)$ & $36,9(33,6-40,3)$ & \\
\hline Mittel & $45,4(44,2-46,7)$ & $46,0(44,7-47,3)$ & $39,8(36,8-43,0)$ & \\
\hline Hoch & $27,1(25,4-28,8)$ & $27,5(25,7-29,3)$ & $23,3(20,4-26,4)$ & \\
\hline Geschwisteranzahl & & & & 0,091 \\
\hline Einzelkind & $19,1(18,1-20,2)$ & $19,0(18,0-20,1)$ & $22,5(18,3-27,3)$ & \\
\hline $1-2$ & $70,3(69,2-71,4)$ & $70,5(69,4-71,5)$ & $64,5(59,0-69,8)$ & \\
\hline$\geq 3$ & $10,6(9,9-11,3)$ & $10,5(9,8-11,2)$ & $12,9(9,4-17,6)$ & \\
\hline Alter der Mutter (Jahre) & & & & 0,006 \\
\hline Zur Geburt des Kindes $<20$ & $2,1(1,9-2,4)$ & $2,1(1,9-2,4)$ & $3,2(1,7-5,7)$ & \\
\hline $20-25$ & $16,6(15,6-17,7)$ & $16,7(15,7-17,8)$ & $15,2(10,3-21,8)$ & \\
\hline $26-30$ & $34,7(33,8-35,7)$ & $34,9(34,0-35,9)$ & $27,2(22,6-32,4)$ & \\
\hline$>30$ & $46,5(45,0-47,9)$ & $46,3(44,8-47,7)$ & $54,5(46,0-62,6)$ & \\
\hline Migrationshintergrund & & & & $<0,001$ \\
\hline $\mathrm{Ja}$ & $17,1(15,2-19,2)$ & $15,3(13,5-17,3)$ & $34,1(30,1-38,3)$ & \\
\hline Nein & $82,9(80,8-84,8)$ & $84,7(82,7-86,5)$ & $65,9(61,7-69,9)$ & \\
\hline \multicolumn{4}{|c|}{$\begin{array}{l}\text { Elterliche Einstellung zu Impfungen (Eltern gaben als Grund gegen eine oder mehrere } \\
\text { Impfungen an, dass sie das Durchmachen einiger Erkrankungen für besser hielten) }\end{array}$} & $<0,001$ \\
\hline Nein & $95,0(94,5-95,6)$ & $95,4(94,8-95,9)$ & $91,4(89,5-93,0)$ & \\
\hline Ja & $5,0(4,4-5,5)$ & $4,6(4,1-5,2)$ & $8,6(7,0-10,5)$ & \\
\hline
\end{tabular}

hängig voneinander mit einer höheren Wahrscheinlichkeit verbunden, dass Eltern Impfnebenwirkungen in der Befragung angaben. Die stärkste diesbezügliche Determinante - die zudem mit einer geringen Irrtumswahrscheinlichkeit statistisch abgesichert werden konnte - war eine kritische elterliche Impfeinstellung: Eltern, die eine oder mehrere Impfungen nicht hatten geben lassen, weil sie es für besser hielten, dass ihr Kind die jeweilige Erkrankung selbst durchmacht, gaben mehr als dreimal so häufig Impfnebenwirkungen an als Eltern, die diese Einstellung nicht hatten (Odds Ratio: 3,29; 95\%KI 2,28-4,75) (• Tab. 2).

\section{Abhängigkeit der Neben- wirkungsberichte vom zeitlichen Abstand zur Impfung}

- Tab. 3 zeigt die Ergebnisse der uniund multivariaten logistischen Regression zum Zusammenhang zwischen der Angabe einer Nebenwirkung nach Masernimpfung mit soziodemografischen Variablen sowie abhängig vom zeitlichen Abstand zur letzten Impfung. Die Wahrscheinlichkeit, dass Eltern innerhalb eines Jahres nach der ersten Masernimpfung eine Impfnebenwirkung angaben, war fast dreimal höher als nach Ab- lauf eines Jahres (OR: 2,88; 95\%-KI 1,33$6,24)$. Auch hier bestand ein starker $\mathrm{Zu}$ sammenhang zur elterlichen Impfeinstellung (OR: 4,12; 95\%-KI 1,62-10,48). Auch die Modellierung des Zusammenhangs zwischen berichteten Masernimpfnebenwirkungen und dem zeitlichen Abstand zur letzten Masernimpfung ergab einen signifikanten Zusammenhang (OR: 2,14; 95\%-KI 1,17-3,92).

\section{Diskussion}

Die Untersuchung von Determinanten für die Angabe einer Impfnebenwirkung erfolgte hypothesengesteuert für Faktoren, die assoziiert sein könnten mit der Einstellung zu Impfungen, mit der Aufmerksamkeit gegenüber beziehungsweise der Bewertung von Gesundheitsstörungen als potenzielle Nebenwirkung einer vorangegangenen Impfung, mit einer möglicherweise erhöhten Vulnerabilität, mit dem zeitlichen Abstand zwischen Impfung(en) und Untersuchungstermin und mit der Anzahl verabreichter Impfungen.

Die stärkste und stabilste Determinante war die Elternangabe, dass sie (eine oder einige) Impfungen nicht durchgeführt hätten, weil sie es für besser hielten, dass ihr Kind einige Erkrankungen selbst durchmacht. Diese Begründung wurde als Hinweis auf eine impfkritische Haltung der Eltern gewertet. Allerdings ist wegen des Querschnittdesigns der Studie nicht zu beurteilen, ob die (retrospektive) Erhebung von Impfnebenwirkungen durch eine bereits bestehende kritische Einstellung zu Impfungen beeinflusst ist oder ob möglicherweise aufgetretene Impfnebenwirkungen erst zu einer impfkritischen Einstellung der Eltern geführt oder dazu beigetragen haben.

Interessanterweise ist bei der Untersuchung von Determinanten für die Angabe von Impfnebenwirkungen dann kein Zusammenhang mit dem sozioökonomischen Status mehr nachzuweisen, wenn im logistischen Modell die Impfeinstellung der Eltern berücksichtigt wird. Ein ähnlicher Zusammenhang konnte bereits bei den Determinanten für Masernimpfungen gezeigt werden [14]. Dies ist so zu interpretieren, dass der Zusammenhang „Angabe von Impfnebenwirkungen/kri- 
tische elterliche Impfeinstellung" stärker ist als der Zusammenhang „Angabe von Impfnebenwirkungen/hoher sozialer Status“. Von Eltern mit einem hohen sozialen Status werden jedoch kritische Impfeinstellungen häufiger vertreten.

Für Kinder und Jugendliche mit Migrationshintergrund wurden seltener Nebenwirkungen nach Impfungen berichtet als für Kinder ohne Migrationshintergrund. Allerdings zeigte sich dieser Zusammenhang nur in der univariaten Auswertung als statistisch signifikant. Da für einen relativ hohen Anteil an Kindern mit Migrationshintergrund die Variable des CSHCN-Screeners nicht erhoben werden konnte, sind sie im Endmodell nicht mehr vollständig repräsentiert [15]. Eine mögliche Erklärung dafür, dass Eltern von Kindern mit Migrationshintergrund seltener Impfnebenwirkungen angaben, könnte eine andere Einstellung zu Impfungen oder Beschwerden nach Impfungen sein. So geben Eltern von Kindern mit Migrationshintergrund als Grund für die Ablehnung von Impfungen seltener Angst vor Nebenwirkungen an [16]. Eine entsprechende Erklärung könnte auch für die Beobachtung, dass Impfnebenwirkungen in den neuen Bundesländern seltener berichtet wurden, vermutet werden. Auswertungen zur Häufigkeit unerwünschter Arzneimittelnebenwirkungen, die sich auf die letzten sieben Tage bezogen, ergaben keine Unterschiede zwischen alten und neuen Bundesländern [17].

Der in der univariaten Auswertung beobachtete Zusammenhang zwischen dem Alter des Kindes und der Wahrscheinlichkeit einer elterlichen Angabe von Impfnebenwirkungen stellte sich in der multivariaten Analyse als nicht statistisch signifikant heraus. Allerdings zeigte sich eine deutlich höhere Prävalenz von Impfnebenwirkungen in der Altersgruppe der Sieben- bis Zehnjährigen. Für diese Altersgruppe wurden doppelt so häufig Impfnebenwirkungen angegeben als für die Gruppe der Drei- bis Sechsjährigen. Ein möglicher Grund für die höhere Prävalenz von Angaben zu Impfnebenwirkungen bei Sieben- bis Zehnjährigen könnte eine höhere kumulative Gesamtanzahl an erhal-

Tab. 2 Uni- und multivariate Odds Ratios (OR) für den Zusammenhang zwischen soziodemografischen und gesundheitsbezogenen Variablen und der Angabe von Impfnebenwirkungen, $\mathrm{n}=15.958$

\begin{tabular}{|c|c|c|c|c|c|}
\hline & \multicolumn{2}{|c|}{ Impfnebenwirkung angegeben } & \multirow[b]{2}{*}{ p-Wert } & \multirow[b]{2}{*}{$\begin{array}{l}\text { Adjustiertes } O^{\mathrm{a}} \\
(95 \%-<\mathrm{KI})\end{array}$} & \multirow[b]{2}{*}{ p-Wert } \\
\hline & $\%(95 \%-K I)$ & $\begin{array}{l}\text { Univariates OR } \\
(95 \%-\mathrm{KI})\end{array}$ & & & \\
\hline Gesamt $(n=332)$ & \multicolumn{2}{|l|}{$2,1(1,8-2,5)$} & & & \\
\hline \multicolumn{2}{|l|}{ Geschlecht } & & \multicolumn{2}{|l|}{0,64} & 0,128 \\
\hline Mädchen & $1,9(1,5-2,3)$ & Referenz & & \multicolumn{2}{|l|}{ Referenz } \\
\hline Junge & $2,4(1,9-3,0)$ & $1,27(0,99-1,64)$ & & \multicolumn{2}{|l|}{$1,23(0,94-1,60)$} \\
\hline \multicolumn{2}{|l|}{ Alter } & & \multicolumn{2}{|l|}{$<0,01$} & 0,066 \\
\hline $14-17$ & $2,0(1,5-2,7)$ & $1,39(0,96-2,04)$ & & \multicolumn{2}{|l|}{$1,36(0,91-2,04)$} \\
\hline $11-13$ & $2,3(1,7-3,2)$ & $1,63(1,06-2,49)$ & & \multicolumn{2}{|l|}{$1,37(0,85-2,21)$} \\
\hline $7-10$ & $2,8(2,2-3,6)$ & $2,00(1,38-2,90)$ & & \multicolumn{2}{|l|}{$1,81(1,23-2,68)$} \\
\hline $3-6$ & $1,4(1,0-2,0)$ & Referenz & & \multicolumn{2}{|l|}{ Referenz } \\
\hline $0-2$ & $2,1(1,5-2,9)$ & $1,47(0,99-2,17)$ & & \multicolumn{2}{|l|}{$1,48(0,99-2,23)$} \\
\hline \multicolumn{2}{|l|}{ Region } & & \multicolumn{2}{|l|}{0,01} & 0,018 \\
\hline Ost & $1,4(1,1-1,9)$ & Referenz & & \multicolumn{2}{|l|}{ Referenz } \\
\hline West & $2,3(1,9-2,8)$ & $1,61(1,12-2,31)$ & & \multicolumn{2}{|l|}{$1,61(1,08-2,39)$} \\
\hline \multicolumn{3}{|l|}{ Sozialstatus } & $<0,01$ & & 0,152 \\
\hline Niedrig & $1,6(1,1-2,1)$ & Referenz & & \multicolumn{2}{|l|}{ Referenz } \\
\hline Mittel & $2,0(1,6-2,5)$ & $1,28(0,91-1,78)$ & & \multicolumn{2}{|l|}{$1,08(0,76-1,55)$} \\
\hline Hoch & $3,0(2,3-3,8)$ & $1,94(1,33-2,83)$ & & \multicolumn{2}{|l|}{$1,49(0,95-2,34)$} \\
\hline \multicolumn{2}{|l|}{ Geschwisteranzahl } & 0,36 & & & \\
\hline Einzelkind & $2,5(1,9-3,3)$ & $1,23(0,93-1,63)$ & & & \\
\hline $1-2$ & $2,1(1,7-2,5)$ & Referenz & & & \\
\hline$\geq 3$ & $2,2(1,5-3,3)$ & $1,08(0,70-1,65)$ & & & \\
\hline Alter der Mutter (Jahre) & & & 0,034 & & 0,563 \\
\hline$<20$ & $1,7(0,8-3,4)$ & $1,12(0,52-2,41)$ & & $1,00(0,42-2,43)$ & \\
\hline $20-25$ & $1,5(1,1-2,1)$ & Referenz & & Referenz & \\
\hline $26-30$ & $2,4(1,9-3,0)$ & $1,62(1,16-2,25)$ & & $1,17(0,82-1,67)$ & \\
\hline$>30$ & $2,2(1,8-2,8)$ & $1,49(1,01-2,19)$ & & $0,96(0,62-1,48)$ & \\
\hline Migrationshintergrund & & & 0,003 & & 0,215 \\
\hline Ja & $1,2(0,8-1,8)$ & Referenz & & Referenz & \\
\hline Nein & $2,3(1,9-2,8)$ & $1,99(1,28-3,13)$ & & $1,41(0,82-2,42)$ & \\
\hline $\begin{array}{l}\text { Children with Special } \\
\text { Health Care Needs } \\
\text { (CSHCN) }\end{array}$ & & & 0,006 & & 0,015 \\
\hline Ja & $3,1(2,3-4,2)$ & $1,52(1,13-2,04)$ & & $1,49(1,08-2,04)$ & \\
\hline Nein & $2,1(1,7-2,5)$ & Referenz & & Referenz & \\
\hline $\begin{array}{l}\text { Elterliche Einstellung zu } \\
\text { gegen eine oder mehrer } \\
\text { machen einiger Erkrank }\end{array}$ & $\begin{array}{l}\text { npfungen (Elte } \\
\text { Impfungen an } \\
\text { ggen für besse }\end{array}$ & $\begin{array}{l}\text { gaben als Grund } \\
\text { ass sie das Durch- } \\
\text { ielten) }\end{array}$ & $<0,001$ & & $<0,001$ \\
\hline Nein & $1,9(1,5-2,2)$ & Referenz & & Referenz & \\
\hline Ja & $6,9(5,1-9,2)$ & $3,92(2,80-5,49)$ & & $3,29(2,28-4,75)$ & \\
\hline $\begin{array}{l}\text { alns Modell aufgenommen } \\
\text { signifikant mit der Angabe } \\
\text { Geschlecht. Erklärte Varianz }\end{array}$ & $\begin{array}{l}\text { urden die oben } \\
\text { Impfnebenwi } \\
\text { ch Nagelkerke: }\end{array}$ & $\begin{array}{l}\text { zeigten Variablen, die } \\
\text { Ingen assoziiert ware } \\
\text { 35. }\end{array}$ & $\begin{array}{l}\text { univariat } \\
\text { ר. Zusätzli }\end{array}$ & $\begin{array}{l}\text { uf dem 5\%-Niveau sta } \\
\text { n erfolgte die Adjustier }\end{array}$ & $\begin{array}{l}\text { tistisch } \\
\text { ung für }\end{array}$ \\
\hline
\end{tabular}

tenen Impfungen in dieser Gruppe aufgrund ihres höheren Lebensalters sein; und die Variable "Alter" würde eine Hilfsvariable für die Anzahl der verabreichten Impfungen darstellen. Die Anzahl der Impfungen war trotz der Daten- erhebung auf Basis der Impfdokumente nicht bestimmbar, weil für viele Impfungen (insbesondere in älteren Impfdokumenten) nicht ersichtlich war, ob sie mit Mono- oder mit Kombinationspräparaten durchgeführt worden waren. 
Tab. 3 Uni- und multivariate Odds Ratios (OR) für den Zusammenhang zwischen soziodemografischen und gesundheitsbezogenen Variablen und der Angabe von Impfnebenwirkungen nach einer Masernimpfung in der Untergruppe Masern-geimpfter Kinder und Jugendlicher, $n=14.171$

\section{Nebenwirkung nach Masernimpfung \\ $\%(95 \%-\mathrm{KI})$ \\ Univariates $\mathrm{OR}$ \\ (95\%-KI)}

Gesamt $(n=63)$

$0,4(0,3-0,6)$

Geschlecht

Mädchen

Junge

$0,3(0,2-0,6)$

Referenz

$0,5(0,3-0,7)$

$1,39(0,83-2,33)$

Elterliche Einstellung zu Impfungen (Eltern gaben als

Grund gegen eine oder mehrere Impfungen an, dass

sie das Durchmachen einiger Erkrankungen für besser

hielten)

Nein

Ja

Abstand zur ersten

$0,4(0,3-0,6)$

Referenz

$1,6(0,7-4,0)$

$4,27(1,75-10,43)$

0,004

Referenz

$4,12(1,62-10,48)$

Referenz

$1,35(0,79-2,30)$

0,002

0,003

Masernimpfung

$<1$ Jahr

$1,1(0,5-2,3)$

$3,02(1,44-6,33)$

$>1 \mathrm{Jahr} \quad 0,4(0,3-0,5) \quad$ Referenz

ans Modell aufgenommen wurden die oben gezeigten Variablen, die univariat auf dem 5\%-Niveau statistisch signifikant mit der Angabe von Impfnebenwirkungen assoziiert waren. Zusätzlich erfolgte die Adjustierung für Geschlecht. Erklärte Varianz nach Nagelkerke: 0,02.

Angesichts veränderter STIKO-Empfehlungen zu Standardimpfungen sowie aufgrund der zunehmenden Anwendung von Kombinationspräparaten ist die Interpretation der beobachteten Zusammenhänge zwischen dem Alter des Kindes und der Angabe von Impfnebenwirkungen schwierig. Zusätzlich wird die Interpretation dadurch erschwert, dass Sieben- bis Zehnjährige mehr Impfungen erhalten haben könnten, für die häufiger von Nebenwirkungen berichtet wurden. So sind Kinder der älteren Altersgruppe deutlich häufiger gegen FSME (19,6\%; 95\%-KI 16,423,2 versus 12,6; 95\%-KI 9,9-15,8) und deutlich häufiger mit BCG (30,8\%; 95\%KI 26,8-35,1 versus 4,6\%; 95\%-KI 3,6$6,0)$ geimpft worden als jüngere Kinder, und die erste Zulassung eines azellulären und damit nebenwirkungsärmeren Pertussisimpfstoffes erfolgte erst 1995. Ältere Kinder können zudem auch noch mit einem FSME-Impfpräparat geimpft worden sein, das 2001 wegen häufigen Auftretens von Nebenwirkungen vom Markt genommen wurde. Bei den Nebenwirkungsberichten wurde jedenfalls in der Gruppe der Sieben- bis Zehnjährigen häufiger über Nebenwirkungen einer FSME-Impfung berichtet als in der Gruppe der Drei- bis Sechsjährigen. Angesichts der insgesamt geringen Anzahl von Nebenwirkungen sollte eine Interpretation des beobachteten Unterschieds nach dem Alter jedoch zurückhaltend erfolgen.

Für Kinder mit einem speziellen Versorgungsbedarf aufgrund chronischer Gesundheits-, Entwicklungs- oder Verhaltensstörungen (CSHCN-Screener positiv) wurden häufiger Nebenwirkungen angegeben als für Kinder ohne solche Probleme. Ursache hierfür könnte eine höhere Vulnerabilität dieser Gruppe aufgrund ihrer chronischen Gesundheitsstörungen sein, zudem kann angenommen werden, dass die Eltern diese Kinder aufmerksamer beobachten.

Die Untersuchung des Zusammenhangs zwischen der elterlichen Angabe einer Impfnebenwirkung und dem zeitlichen Abstand zwischen Impfung und Befragung wird in unserer Studie durch mehrere Faktoren erschwert. So konnte die Zuordnung der berichteten Nebenwirkungen zu einer bestimmten Impfung nicht mit hinreichender Genauigkeit erfolgen. Zwar wurde im Interview danach gefragt, jedoch nicht so differenziert, dass in jedem Falle eine eindeutige Zuordnung zwischen Nebenwir- kung und Impfung möglich war. Außerdem erinnerten sich viele Eltern nicht an die Impfung, nach der eine Nebenwirkung aufgetreten war. Um detailliert $\mathrm{zu}$ untersuchen, ob die Länge des zwischen Impfung und Erhebung vergangenen Zeitraumes die elterlichen Angaben zu aufgetretenen Nebenwirkungen beeinflusst (Erinnerungsbias), könnte man bei allen Befragten den zeitlichen Abstand des Erhebungszeitpunktes zur ersten verabreichten Impfung oder auch zur letzten Impfung ermitteln und mit analysieren. Allerdings wird ein solches Vorgehen durch die sehr große Zahl an unterschiedlichen Impfungen bei relativ geringer Zahl an berichteten Nebenwirkungen und vor allem dadurch erschwert, dass viele berichtete Impfnebenwirkungen nicht nach Verabreichung der ersten Impfung und auch nicht nach der der letzten auftraten, sondern bei Impfungen, die dazwischen lagen. Etwas spezifischer lässt sich der mögliche Effekt untersuchen, wenn man die Analyse auf eine Impfung beschränkt, bei der für eine erfolgreiche Immunisierung nur wenige Impfdosen verabreicht werden müssen. Diese Bedingung wird am besten für die Masernimpfung erfüllt: Hier wird zur Immunisierung die Verabreichung von nur zwei Dosen empfohlen.

Erwartungsgemäß kann in unserer Studie gezeigt werden, dass auch die retrospektive Erhebung von Impfnebenwirkungen dem Erinnerungsbias unterliegt. Darüber hinaus scheint die Angabe von Impfnebenwirkungen von Faktoren beeinflusst zu werden, die mit der persönlichen Wahrnehmung und Bewertung möglicher Nebenwirkungen sowie mit der Einstellung zu Impfungen zusammenzuhängen scheinen.

\section{Korrespondenzadresse \\ Dr. C. Poethko-Müller}

Abteilung für Epidemiologie und Gesundheitsberichterstattung, Fachgebiet Kinder- und Jugendgesundheit, Prävention, Robert Koch-Institut

Postfach 13302 Berlin

Poethko-MuellerC@rki.de

Interessenkonflikt. Der korrespondierende Autor weist auf folgende Beziehungen hin:

Dr. med. Christina Poethko-Müller MSc: Koordinierung einer gemeinsam durch das Bundesministerium für 
Gesundheit, das Paul-Ehrlich-Institut, Sanofi Pasteur und GlaxoSmithKline finanzierten epidemiologischen Studie über Todesfälle bei Kindern im 2. bis 24. Lebensmonat (TOKEN-Studie) in den Jahren 2004-2009. PD Dr. med. Martin Schlaud: Studienleitung einer gemeinsam durch das Bundesministerium für Gesundheit, das Paul-Ehrlich-Institut, Sanofi Pasteur und GlaxoSmithKline finanzierten epidemiologischen Studie über Todesfälle bei Kindern im 2. bis 24. Lebensmonat (TOKEN-Studie) in den Jahren 2004-2009.

Dr. med. Karen Atzpodien und Dr. oec. troph. Roma Schmitz: Es besteht kein Interessenskonflikt der Autorinnen.

\section{Literatur}

1. Chen RT (1999) Vaccine risks: real, perceived and unknown. Vaccine 17(Suppl 3):S41-S46

2. Rosenthal KL, McVittie LD (1993) The clinical testing of preventive vaccines. In: Mathieu M (Hrsg) Biologic development: a regulatory overview. Paraxel, Waltham, MA, S. 119-130

3. Schneeweiß B, Pfleiderer M, Keller-Stanislawski B (2008) Impfsicherheit heute. Dtsch Ärztebl 105(34-35):590-595

4. Griffin MR, Braun MM, Bart KJ (2009) What should an ideal vaccine postlicensure safety system be? Am J Public Health 99(Suppl 2):S345-S450

5. Blenkinsopp A, Wilkie P, Wang M, Routledge PA (2007) Patient reporting of suspected adverse drug reactions: a review of published literature and international experience. Br J Clin Pharmacol 63(2):148-156

6. Hunsel F van, Passier A, Grootheest K van (2009) Comparing patients' and healthcare professionals' ADR reports after media attention: the broadcast of a Dutch television programme about the benefits and risks of statins as an example. $\mathrm{Br}$ J Clin Pharmacol 67(5):558-564

7. Lopez-Gonzalez E, Herdeiro MT, Figueiras A (2009) Determinants of under-reporting of adverse drug reactions: a systematic review. Drug Saf 32(1):19-31

8. Hölling H, Kamtsiuris P, Lange M et al (2007) Der Kinder- und Jugendgesundheitssurvey (KiGGS): Studienmanagement und Durchführung der Feldarbeit. Bundesgesundheitsbl Gesundheitsforsch Gesundheitsschutz 50(5-6):557-566

9. Kurth B-M (2007) Der Kinder- und Jugendgesundheitssurvey (KiGGS): Ein Überblick über Planung, Durchführung und Ergebnisse unter Berücksichtigung von Aspekten eines Qualitätsmanagements. Bundesgesundheitsbl Gesundheitsforsch Gesundheitsschutz 50(5-6):533-546

10. Kurth B-M, Kamtsiuris $\mathrm{P}$, Hölling $\mathrm{H}$ et al (2008) The challenge of comprehensively mapping children's health in a nation-wide health survey: design and first results of the German KiGGS-Study. BMC Public Health 8:196

11. Lange $M$, Kamtsiuris $P$, Lange $C$ et al (2007) Messung soziodemographischer Merkmale im Kinder- und Jugendgesundheitssurvey (KiGGS) und ihre Bedeutung am Beispiel der Einschätzung des allgemeinen Gesundheitszustands. Bundesgesundheitsbl Gesundheitsforsch Gesundheitsschutz 50(5-6):578-589

12. Schmidt S, Thyen U, Petersen C, Bullinger M (2004) The performance of the screener to identify children with special health care needs in a European sample of children with chronic conditions. Eur J Pediatr 163(9):517-523
13. Keller-Stanislawski B, Hartmann K (2002) Auswertung der Meldungen von Verdachtsfällen auf Impfkomplikationen nach dem Infektionsschutzgesetz. Bundesgesundheitsbl Gesundheitsforsch Gesundheitsschutz 45:344-354

14. Poethko-Muller C, Ellert U, Kuhnert R et al (2009) Vaccination coverage against measles in Germanborn and foreign-born children and identification of unvaccinated subgroups in Germany. Vaccine 27(19):2563-2569

15. Scheidt-Nave C, Ellert U, Thyen U, Schlaud M (2008) Health care needs of children and adolescents with chronic conditions. Bundesgesundheitsbl Gesundheitsforsch Gesundheitsschutz 51(6):592-601

16. Poethko-Müller C, Ellert U, Kuhnert R et al (2009) Kinder- und Jugendgesundheitsstudie (KiGGS): Impfquoten von Kindern und Jugendlichen mit Migrationshintergrund in Deutschland. Bundeszentrale für gesundheitliche Aufklärung, Köln

17. Knopf H, Du Y (2010) Perceived adverse drug reactions among non-institutionalized children and adolescents in Germany. Br J Clin Pharmacol 70(3):409-417 